\title{
Pseudogap phase formation in the crossover from Bose-Einstein condensation to BCS superconductivity in low dimensional systems
}

\author{
Valery P. Gusynin ${ }^{1}$, Vadim M. Loktev ${ }^{1}$, Rachel M. Quick ${ }^{2}$ and Sergei G. Sharapov ${ }^{2, \dagger}$ \\ ${ }^{1}$ Bogolyubov Institute for Theoretical Physics, \\ 252143 Kiev, Ukraine \\ ${ }^{2}$ Department of Physics, University of Pretoria, \\ 0002 Pretoria, South Africa
}

\begin{abstract}
A phase diagram for a 2D metal with variable carrier density has been studied using the modulus-phase representation for the order parameter in a fully microscopic treatment. This amounts to splitting the degrees of freedom into neutral fermion and charged boson degrees of freedom. Although true long range order is forbidden in two dimensions, long range order for the neutral fermions is possible since this does not violate any continuous symmetry. The phase fluctuations associated with the charged degrees of freedom destroy long range order in the full system as expected. The presence of the neutral order parameter gives rise to new features in the superconducting condensate formation in low dimensional systems.

The resulting phase diagram contains a new phase which lies above the superconducting (here Berezinskii-Kosterlitz-Thouless) phase and below the normal (Fermi-liquid) phase. We identify this phase with the pseudogap phase observed in underdoped high- $T_{c}$ superconducting compounds above their critical temperature. We also find that the phase diagram persists even in the presence of weak 3-dimensionalisation.
\end{abstract}

$\dagger$ On leave from Bogolyubov Institute for Theoretical Physics of the National Academy of Sciences of Ukraine, 252143 Kiev, Ukraine

Invited paper presented at First International Conference on New Theories, Discoveries, and Applications of Superconductors and Related Materials February 19-24, 1998, Baton Rouge, Louisiana, USA 
The anomalous behavior of the normal state of high-temperature superconductors (HTSC) [1] (including the behavior of the spin susceptibility, resistivity, specific heat and photo-emission spectra) has been recently interpreted in terms of the formation of a pseudogap above the critical temperature, $T_{c}$ [2].

There are many approaches developed to explain this remarkable phenomenon. However we present here the point of view that the pseudogap phenomena is an inherent property of a low dimensional system with a relatively small carrier density.

The formation of a pseudogap phase above $T_{c}$ has been explicitly demonstrated in a very simple model non-relativistic 2D Fermi-system [3] and its extension to the quasi-2D case [4]. The work is based on the peculiarities of the Berezinskii-Kosterlitz-Thouless (BKT) phase formation which is a two stage process. The only thing that one needs to analyze the problem is to choose the appropriate variables.

The simplest model Hamiltonian density for fermions confined to a 2D volume $v$ reads

$$
\mathcal{H}=\psi_{\sigma}^{\dagger}(x)\left(-\frac{\nabla^{2}}{2 m}-\mu\right) \psi_{\sigma}(x)-V \psi_{\uparrow}^{\dagger}(x) \psi_{\downarrow}^{\dagger}(x) \psi_{\downarrow}(x) \psi_{\uparrow}(x),
$$

where $x \equiv \mathbf{r}, \tau$ denotes the position and imaginary time; $\psi_{\sigma}(x)$ is a fermion field, $m$ is the effective fermion mass, $\mu$ is a chemical potential, $V$ is an effective local attraction constant; $\hbar=k_{B}=1$.

The Hubbard-Stratonovich method is applied to write the statistical sum $Z(v, \mu, T)$ as a functional integral over fermi-fields (Nambu spinors) and the auxiliary field $\Phi=V \psi_{\uparrow}^{\dagger} \psi_{\downarrow}^{\dagger}$. Contrary to the usual method for the calculation of $Z$ in $\Phi, \Phi^{*}$ variables, for a $2 \mathrm{D}$ system one must rewrite the order parameter $\Phi(x)$ in terms of its modulus $\rho(x)$ and its phase $\theta(x)$ i.e. $\Phi(x)=\rho(x) \exp [-i \theta(x)]$. This was originally stated by Witten in the context of $2 \mathrm{D}$ quantum field theory [5]. This replacement by modulus-phase variables also demands the replacement $\psi_{\sigma}(x)=\chi_{\sigma}(x) \exp [i \theta(x) / 2]$. From a physical point of view this entails replacing the charged fermion particle $\psi_{\sigma}(x)$ by a neutral fermion $\chi_{\sigma}(x)$ and a spinless charged boson $\exp [i \theta(x) / 2]$.

As a result one obtains

$$
Z(v, \mu, T)=\int \rho \mathcal{D} \rho \mathcal{D} \theta \exp [-\beta \Omega(v, \mu, T, \rho(x), \partial \theta(x))]
$$

where

$$
\beta \Omega(v, \mu, T, \rho(x), \partial \theta(x))=\frac{1}{V} \int_{0}^{\beta} d \tau \int d \mathbf{r} \rho^{2}(x)-\operatorname{Tr} \operatorname{Ln} G^{-1}
$$


is the one-loop effective action, which depends on the modulus-phase variables. The action (3) is expressed through the Green function of the initial (charged) fermions written in the new variables.

It is clearly impossible to obtain $\Phi \equiv\langle\Phi(x)\rangle \neq 0$ at finite $T$ since this corresponds to the formation of homogeneous long-range (superconducting) order which is forbidden by the Coleman-Mermin-Wagner-Hohenberg theorem. However it is possible to obtain $\rho \equiv\langle\rho(x)\rangle \neq 0$ but at the same time $\Phi=\rho\langle\exp [-i \theta(x)]\rangle=0$ due to random fluctuations in the phase $\theta(x)$. We stress that $\rho \neq 0$ does not imply long-range superconducting order (which is destroyed by the phase fluctuations) and is therefore not in contradiction with the above-mentioned theorem.

The effective action (3) can be approximately represented in the following form

$$
\Omega(v, \mu, T, \rho, \partial \theta(x))=\frac{T}{2} \int_{0}^{\beta} d \tau \int d \mathbf{r} J(\mu, T, \rho(\mu, T))(\nabla \theta)^{2}+\Omega_{p o t}(v, \mu, T, \rho) .
$$

The kinetic part of the action coincides with the classical XY-model, where now however, $J$ depends on $\mu$ and $\rho$. This allows to use the well-known equation for the BKT temperature: $T_{\mathrm{BKT}}=\frac{\pi}{2} J\left(\mu, T_{\mathrm{BKT}}, \rho\left(\mu, T_{\mathrm{BKT}}\right)\right)$, which should be solved together with the equations for $\mu$ and $\rho$. These equations are obtained from the effective potential $\Omega_{p o t}(v, \mu, T, \rho)$.

Our investigation shows three regions in the 2D phase diagram [3]. The first is the superconducting $(\mathrm{BKT})$ phase, where $T<T_{\mathrm{BKT}}$ and $\rho \neq 0$. In this region there is algebraic order and a power law decay of the superconducting correlations. We note that for small carrier densities (underdoped case) $T_{\mathrm{BKT}}=\epsilon_{F} / 8$ where $\epsilon_{F}$ is the Fermi energy. The second is the pseudogap phase $\left(T_{\mathrm{BKT}}<T<T_{\rho}\right)$ where $\rho$ is still non-zero but the correlations decay exponentially. The temperature $T_{\rho}$ is that at which $\rho$ becomes zero and it is interpreted as the temperature at which the pseudogap opens. There is no phase transition at this temperature. The third is the normal (Fermi-liquid) phase where $\rho=0$. Note that $\Phi=0$ everywhere. The unusual properties of the second region, which lies between the superconducting and normal phases, can be used to explain the pseudogap behavior in HTSC. For example in the mean-field calculation of the paramagnetic susceptibility the parameter $\rho$ plays the role of the energy gap $\Delta$ in the theory of convenient superconductors. Moreover, the full fermion Green function has a branch cut and not a pole above $T_{\mathrm{BKT}}$ which is also in agreement with the available data about the pseudogap.

It is important that all $2 \mathrm{D}$ results obtained persist in the quasi-2D case. In this case one also has a phase transition to a phase with long range order. However the transition temperature, 
$T_{c}$, always lies below the temperature $T_{\mathrm{BKT}}$ for the quasi-2D system or coincides with $T_{\mathrm{BKT}}$ [ [0] . As one expects, in the high density limit, all these temperatures tend asymptotically to the BCS temperature.

Note finally that the description of the phase fluctuations and the BKT transition closely resembles that given by Emery and Kivelson [6]. However in their phenomenological approach the field $\rho(x)$ does not appear while in the present microscopic approach based on the modulusphase representation [5] it appears rather naturally.

\section{Acknowledgements}

We would like to thank Drs. E.V. Gorbar, N.J. Davidson, I.A. Shovkovy and V.M. Turkowskii for numerous discussions. R.M.Q and S.G.Sh also acknowledge the financial support of the Foundation for Research Development, Pretoria.

\section{References}

[1] V.M. Loktev, Sov. Low Temp. Phys. 22, 1 (1996).

[2] M. Randeria, Varenna Lectures (1997), Preprint cond-mat/9710223.

[3] V.P. Gusynin, V.M. Loktev, S.G. Sharapov, JETP Lett. 65, 182 (1997) and submitted to Phys.Rev. B (preprint cond-mat/9709034).

[4] R.M. Quick, S.G. Sharapov, to be published in Physica C (preprint cond-mat/9708151); V.M. Loktev, R.M. Quick, S.G. Sharapov, preprint cond-mat/9804026.

[5] E. Witten, Nucl. Phys. B145, 110 (1978).

[6] V. Emery, S.A. Kivelson, Nature 374, 434 (1995); Phys. Rev. Lett. 74, 3253 (1995). 